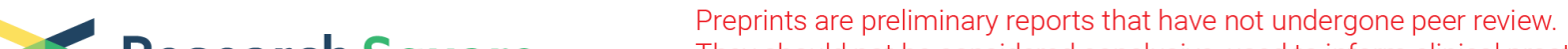 Research Square They should not be considered conclusive, used to inform clinical practice, or referenced by the media as validated information.
}

\section{Self-monitoring of blood pressure to improve hypertension management in a middle-income country: A proof of concept study}

\section{Christelle Akl}

American University of Beirut

Nadine Rashidi

American University of Beirut

Chaza Akik

American University of Beirut Faculty of Health Sciences https://orcid.org/0000-0002-0654-8750

Eveline Hitti

American University of Beirut Medical Center

Ghassan Hamadeh

American University of Beirut Medical Center

Kamal Badr

American University of Beirut Medical Center

\section{Maya Romani}

American University of Beirut Medical Center

\section{Samir Mallat}

American University of Beirut Medical Center

\section{Ali Ghassani}

Amal Association

\section{Serop Ohanian}

Howard Karagheusian Commemorative Center

\section{Fawzi Wafaie}

Harvard University T H Chan School of Public Health

\section{Hala Ghattas}

American University of Beirut

Carla Makhlouf Obermeyer ( $\square$ carlaobermeyer@gmail.com )

\section{Research article}

Keywords: Hypertension management, self-monitoring of blood pressure, systolic blood pressure, diastolic blood pressure, health education, low- and middle- income country, Arab world 
Posted Date: June 4th, 2020

DOl: https://doi.org/10.21203/rs.3.rs-25446/v1

License: (c) (i) This work is licensed under a Creative Commons Attribution 4.0 International License. Read Full License 


\section{Abstract \\ Background}

Self-monitoring of blood pressure has been shown to optimize the management of blood pressure in high-income settings, but there is less evidence from low-to-middle income countries. We designed a proof-of-concept pilot trial to assess the feasibility and acceptability of an intervention built around selfmonitoring of blood pressure and health education, and to measure its association with reduced blood pressure among hypertensives. The study was conducted in Lebanon, a country where the management of hypertension presents challenges similar to those faced in countries of the Arab region and other middle-income countries.

\section{Methods}

We conducted a parallel two-arm pilot trial with a mixed-method approach to investigate the effect of the intervention on patient experience. Hypertensive patients $(n=80)$ were recruited at two primary and one tertiary health centers in Beirut, and were randomly allocated (1:1) to either an intervention group where patients received blood pressure devices, diaries and educational sessions, or a control group where patients received standard of care as practiced in their health centers. The main outcomes were feasibility (recruitment, retention and adherence), acceptability, and changes in systolic blood pressure. Quantitative and qualitative data were obtained at baseline and 6 weeks later.

\section{Results}

The recruitment rates for the study was $52 \%$ and retention was $95 \%$. Most participants in the selfmonitoring group (33/38) reported that the device was convenient and easy to use. Complete case analysis showed that blood pressure monitoring was associated with a greater reduction in systolic $(-6.3 \mathrm{mmHg}, 95 \% \mathrm{Cl}[-12.4 ;-0.17])$ and diastolic $(-1.9 \mathrm{mmHg}, 95 \% \mathrm{Cl}[-6.34 ; 2.58])$ blood pressure in the self-monitoring group $(n=36)$ as compared to the standard of care group $(n=36)$. Improved knowledge of hypertension was also observed in the self-monitoring group. There were no adverse events related to study participation.

\section{Conclusions}

Self-monitoring is acceptable and feasible and has the potential to improve hypertension management. Our results should be further tested in trials with adequate statistical power and longer follow-up periods to examine the effectiveness of the intervention on blood pressure levels.

\section{Trial Registration and funding:}


Retrospectively Registered on April 3, 2020. ISRCTN 16450193. Funded by the Harvard Medical School Center for Global Health Delivery.

\section{Background}

High blood pressure is the leading risk factor for global mortality and a major cause of ischemic heart diseases and stroke (1-4). Hypertension affects $33 \%$ of the population in the Middle East and $29 \%$ of the Lebanese population $(5,6)$. Global data show that control of blood pressure is poor (7-9) especially in low- and middle-income countries (LMICs) with a recent review indicating that only $10 \%$ of hypertensive adults in LMICs have their blood pressure under control (10). In Arab countries, less than third of hypertensive patients have controlled blood pressure, according to a recent review (11), and in Lebanon, a middle-income country of the Arab region (12), control rates are suboptimal and comparable to the regional average $(10,13,14)$.

The growing accessibility of mobile devices and wearable technologies has increased interest in selfmonitoring of blood pressure. Evidence on the effect of self-monitoring is available for high-income countries (15-19), where self-monitoring has the potential to reduce blood pressure levels and improve the medical management of hypertension when compared to usual care $(15,16)$ by engaging patients and motivating them to follow healthy lifestyles $(20,21)$. Recent studies recommend that self-monitoring be part of multi-pronged interventions that include medication titration, education, and lifestyle counseling (22). While it is clear that self-monitoring can optimize the management of blood pressure in high-income settings, there is less evidence from low- and middle-income countries $(23,24)$ and some doubts are expressed about its utility in such settings (25).

The considerable gap in delivering prevention and treatment interventions in Arab countries, and in lowand middle-income countries more generally, underscores the importance of exploring different approaches to the management of blood pressure including engaging patients in the management of their condition. We undertook a project to investigate the feasibility, acceptability, and potential impact of self-monitoring of blood pressure. The project was conducted in Lebanon, a country characterized by population diversity and disparate levels of medicalization, and where efforts have been made to formulate policies and programs to address hypertension and cardiovascular disease (26). As some of these characteristics are shared with other countries of the region and with low-and-middle income countries more generally, the results have relevance beyond the particular setting of the study.

We designed a pilot study to assess the feasibility and acceptability of an intervention built around selfmonitoring of blood pressure and health education. We also examined whether self-monitoring and education about hypertension can contribute to reducing blood pressure levels through (1) increasing knowledge about hypertension management and self-monitoring of blood pressure; (2) improving medication regimen and medication adherence; and (3) fostering healthy behaviors among hypertensive patients. To our knowledge, this is the first study to examine the proof-of-concept of such an intervention in a country of the Middle East and North Africa region. 


\section{Methods}

Study design and overview

This was a mixed-method, parallel two-arm pilot study to explore the factors that need to be considered when implementing an intervention that includes self-monitoring of blood pressure and health education; and assessing its possible effect on patient outcomes. In addition to providing patients with knowledge and devices to monitor their blood pressure and conducting interviews at several points in time, we paid special attention to the design of instruments and the production of health education materials and carefully adapted them to the socio-cultural context, building on a previously conducted situation analysis of hypertension in Lebanon. This pilot study followed the CONSORT 2010 extension for randomized pilot and feasibility trials statement (27) (Additional file 1).

Respondent selection

Hypertensive patients were recruited from physicians' waiting rooms in two primary and one tertiary health care centers in Beirut. Patients were eligible if they were Lebanese adults (18+) and had a prior diagnosis of hypertension. Participants were excluded prior to randomization if their spouse was a participant in the study or if they had physical or mental health conditions that would interfere with adequate monitoring of blood pressure. Eligible patients were identified by the clinical team, and then approached by the research team who invited them to participate in the study. All patients who agreed to participate provided written informed consent at the beginning of the study. Recruitment and data collection were carried out between July and September 2018. The study was approved by the Institutional Review Board of the American University of Beirut.

Participant allocation and study intervention

The 80 patients who agreed to participate in the study were randomly and equally allocated (ratio 1:1), based on a computer-generated series of numbers, to either self-monitoring of blood pressure group which includes receiving diaries and educational sessions $\left(n_{1}=40\right)$, or standard of care group $\left(n_{2}=40\right)$. Randomization was stratified by gender and health centers. The project coordinator, who did not participate in data collection or recruitment, generated the randomization lists and put papers with the assigned group inside consecutively numbered and closed opaque envelopes. The data collectors who recruited participants had no access to the randomization lists. They assigned an envelope to each participant who agreed to enter the study. Envelopes were sealed and were only opened after recruitment to ensure allocation concealment.

\section{Intervention or self-monitoring group}

Patients received 1) an Omron MIT Elite Plus arm monitor (Omron Corporation, Schaumburg, IL); 2) a oneon-one educational session using a brochure with infographics on blood pressure and 3) a diary to record blood pressure measurements and their experience with blood pressure and with the monitors. Patients 
were asked to measure and record their blood pressure in the diary following a set schedule, and they were also encouraged to measure it as frequently as they would like. The schedule of the measurements was established in light of trade-offs between obtaining enough measurements and not asking too much of participants. It was defined as follows: during week 1 and week 6 (at baseline and endline) daily, twice a day, morning and evening; and during weeks 2 to 5 : twice a week, on two non-consecutive days (one weekday and one weekend day), once a day. Participants were advised to choose the same days and times to take their blood pressure from week 2 to 5 . A total of 108 blood pressure measurements were requested from the patients for the whole study period. The researcher team called participants at the end of week 1 and at the beginning of week 6 to remind them about the study protocol; as well as at the end of the study to schedule the endline questionnaire. Participants were offered reimbursement for transportation to and from study sites and were considered lost to follow-up if they did not respond to the research team after four call attempts.

Research assistants trained patients in proper use of the home blood pressure devices; they showed them how to take three consecutive measurements for each recording session and how to record blood pressure measurements on the device, and they made sure that patients were able to perform measurements correctly. At endline, all measurements were downloaded from the device to the research computer using the Omron Health Management Software. The diary served as a means to remind participant about the study protocol and to encourage them to write down their feelings and experiences with the self-monitoring process.

The educational intervention used a specially designed brochure to provide information about blood pressure and we held a one face-to-face session with participants at baseline to explain the information in the brochure. We used information available on the websites of major professional organizations (28, 29) and created a brochure with infographics that explained hypertension and its causes, gave tips about measurements and monitoring, and provided advice about lifestyle and medications. We adapted the information to the local context, building on a previously conducted situation analysis, which had provided insights into understandings of blood pressure, prevalent approaches to diagnosis and management of hypertension, perceptions of the disease and available therapies. The infographics brochure was produced in English and Arabic using simple visuals in an attractive format.

\section{Control or standard of care group}

Patients received standard hypertension care as defined by the health facility and healthcare provider, which may or may not include blood pressure monitoring. Although they were not given blood pressure monitors or a brochure at recruitment, they received the infographics brochure and an education session at endline.

\section{Data collection}

Face-to-face interviews were conducted with all participants at baseline and six weeks later at endline-a follow-up period that reflects trade-offs between the need for a longitudinal view and the resources 
available. The same questionnaire was used for both groups at baseline; the endline questionnaire includes more questions to patients in the self-monitoring group, about experiences and perceptions around monitoring. For both groups, we collected data on the main outcomes as well as on the background and confounding variables. We used close - ended questions to collect quantifiable data about sociodemographic and clinical variables, medication changes and medication adherence, knowledge about hypertension, and blood pressure measurements; and we used open-ended questions to collect data about experiences, perceptions and opinions. Health literacy was assessed by asking 5 questions adapted from the All Aspects of Health Literacy Scale (30), and body mass index (BMI = $\left.\mathrm{kg} / \mathrm{m}^{2}\right)$ was calculated from self-reported weight $(\mathrm{kg})$ and height $(\mathrm{m})$.

\section{Outcome measures}

In this paper, we focus on those quantifiable responses that are relevant for a proof of concept analysis. We also draw on the qualitative data from the open-ended questions to get insights into patients' experiences and we provide selected quotes to illustrate the findings

\section{Feasibility}

We used three quantifiable variables to assess the feasibility of our intervention: first, recruitment rates; secondly, retention rates (i.e. the percentage of patients from the self-monitoring group who completed the 6-week study); and thirdly, the extent to which participants adhered to the blood pressure measurements protocol. Adherence was assessed based on the measurements downloaded from the devices, by dividing the total observed measurements by the total expected measurements (i.e. 108 measurements). We complemented these quantitative variables by analyzing the texts transcribed from responses to two open-ended questions about whether respondents would continue using the device after the end of the study, and whether they planned to discuss the information provided in the brochure with their acquaintances. We recoded responses into quantifiable variables and used the texts to provide illustrative quotes about participants' responses to the intervention.

\section{Acceptability}

Participants in the self-monitoring group were asked about their experiences with self-monitoring of blood pressure and their opinion of the educational materials. We recoded their responses to obtain a quantifiable measure of the acceptability of the intervention, and we also provide illustrative quotes. Details can be found in Additional file 2 .

\section{Effectiveness}

Since the main goal of the study was to explore the effectiveness of self-monitoring for improving the management of hypertension, we analyzed changes in systolic blood pressure (as primary outcome), in diastolic blood pressure, knowledge scores, medication and lifestyle changes (as secondary outcomes).

\section{Changes in blood pressure level}


The three blood pressure measurements (systolic and diastolic) taken at each study visit were averaged and used to determine blood pressure changes between baseline and endline.

\section{Changes in knowledge score}

The questionnaire administered at baseline and endline included 16 questions designed to test respondents' understanding of the information in the brochure; we analyzed responses and calculated a knowledge score based on accuracy of responses.

\section{Changes in medication adherence and medications}

The 8-item Morisky Medication Adherence Scale (MMAS-8) was used to assess adherence to medications at baseline and endline (31). Response categories were yes/no for each item and a 5-point Likert response for the last item. The total score on the MMAS-8 ranges from 1 to 8 , with scores less than 6 reflecting low adherence $(31,32)$. We also assessed through a close-ended question whether any change in types, numbers, or dosages of prescribed antihypertensive medications occurred over the 6week study period.

\section{Changes in lifestyle habits}

Participants from both self-monitoring of blood pressure and standard of care groups were asked an open-ended question during the endline interview, about whether they made any change in their lifestyle habits over the course of the 6-week study. We quantified and analyzed their reported changes in lifestyle habits, including physical activity levels, salt intake, dietary intake, and weight management.

All the tools and questionnaires that we developed were translated to Arabic and piloted to ensure that they were adequately understood and that they were adapted to the local context.

Sample size and duration of the study

A powered randomized controlled trial would have required a sample of around 600 patients (300 per group, allowing for a 15\% loss to follow-up) to detect a difference of $5 \mathrm{~mm} \mathrm{Hg}$ in change in mean systolic blood pressure over 6 months between the intervention and control groups, with an $80 \%$ power, using a $5 \%$ significance level, and assuming that the standard deviation (SD) was $\pm 20 \mathrm{~mm} \mathrm{Hg}$ (33). Given that the resources for such a trial would be difficult to obtain without preliminary work, we opted for a proofof-concept study, with smaller sample size and shorter follow-up period, which would provide enough variability and suggestive evidence that could be pursued with a larger trial. Thus, a sample of 80 participants and a follow-up of 6 weeks were determined to be adequate in light of trade-offs between the available resources and the goals of the study (34).

\section{Statistical Analysis}

We conducted statistical analyses of the data in order to detect suggestive findings as part of a proof-ofconcept analysis. Descriptive statistics on the study population were conducted and expressed as mean and standard deviation (SD) for normally distributed continuous variables; median and interquartile range 
(IQR) for non-normal continuous variables; and frequency for categorical variables. Independent t-tests and Mann-Whitney tests were used to evaluate the differences between self-monitoring of blood pressure and standard of care groups for continuous variables and chi-squared test were used for categorical variables. As in other studies and despite randomization $(23,35)$, we observed a statistical difference between groups for blood pressure baseline values; consequently, we included these variables as covariates in the multivariate models. Linear and logistic regression models, adjusted for age, sex, baseline blood pressure values, and health centers, were used to estimate differences in changes from baseline between self-monitoring of blood pressure and standard of care for continuous and categorical outcomes, respectively. Blood pressure levels, knowledge scores and MMAS scores were treated as continuous variables; and medication changes and lifestyle improvement (yes/no) were treated as categorical variables. Analyses were based on an intention-to-treat approach whereby individuals were analyzed in the groups to which they were randomized (36). A complete case analysis was conducted and, for the primary outcome, a sensitivity analysis was carried out to take into consideration different assumptions regarding missing data and outliers. In order to gain insights into patient experiences and to explore the protocol's acceptability and feasibility, we analyzed qualitative data collected in response to open-ended questions and illustrated the findings with quotes. Patient responses were tape-recorded, transcribed, translated from Arabic to English, and analyzed in two ways. First, where data could be quantified (i.e. self-reported lifestyle changes) we conducted statistical analyses. Secondly, for data about participants' perceptions and experiences with blood pressure monitoring and hypertension management, transcripts were coded and analyzed by team members trained in qualitative methods and assisted by the computer software Dedoose version 4.0 (Dedoose; SocioCultural Research Consultants LLC). All statistical analyses were carried out using STATA version 13 (STATA Corporation, College Station, Texas, USA) and statistical significance was defined at an alpha level of $5 \%$.

\section{Results}

Socio-demographic and clinical characteristics

Eighty outpatients from the tertiary hospital and primary health care centers were enrolled in the study (Additional file 3). One patient left the study before receiving the intervention due to logistics constraints, yielding a total of 79 baseline interviews. The socio-demographic and clinical characteristics of patients in the two groups are shown in Additional file 4. Just over two thirds of participants (71\%) were recruited from the two primary health care centers and the remaining (29\%) from the hospital. The average age of the participants was $60.2 \pm 9.7$ years. Almost three quarter of participants were from low socio-economic status (73\%). Most participants were married (70\%) and more than half of them were not employed (52\%). Almost half of all study participants were obese $\left(\mathrm{BMI}>30 \mathrm{~kg} / \mathrm{m}^{2}\right)$ and $82 \%$ reported other comorbidities such as diabetes, hypercholesterolemia, hypertriglyceridemia, cardiovascular or kidney diseases. Also, only half of the patients $(49 \%)$ had a blood pressure target to reach.

Although subjects were randomly assigned to either the self-monitoring of blood pressure group or standard of care group, systolic and diastolic blood pressure numbers were significantly higher among 
participants from the standard group. This variable was thus included as an additional control for confounding in regression models. Differences in background variables between groups at baseline including socio-demographic characteristics, health literacy levels, BMI categories, duration of hypertension, number of antihypertensive medications, ownership of a blood pressure device, and frequency of blood pressure monitoring were not significant.

Feasibility

The recruitment rates for the study overall was $52 \%$. It was necessary to approach almost twice as many patients as the targeted number: 152 patients were approached and met the inclusion criteria and 79 consented and were enrolled. The 73 patients who declined to take part in this study reported that they had no time, were not interested, or found it inconvenient to travel to the health center. Non-responders did not differ from responders in terms of age, but a non-significant greater number of females from the tertiary health center refused to participate in the study as compared to men, while the opposite pattern was observed in primary health centers. The recruitment rate was higher at the primary centers $(70 \%)$ as compared to tertiary (31\%) health center, and it was generally easier to recruit participants from low- and middle-income groups. Retention was high, at around $95 \%$; out of the 79 patients enrolled in the study, 75 completed the 6 weeks. Three participants from the standard of care group recruited at the tertiary health center and one from the self-monitoring of blood pressure group recruited at the primary health center did not complete the 6 weeks, and were considered lost to follow-up after they did not respond to the 4 call attempts by the research team for the end line interview. Participants who completed the study were similar to those lost to follow-up in terms of gender and age.

All patients took on average 95 out of the total expected 108 measurements; this represents a level of adherence of $93.9 \% \pm 20 \%$ (median \pm IQR). In our protocol, participants were encouraged to measure their blood pressure as frequently as they would like - outside the required protocol; but few did so as they considered that they were already taking many measurements.

Almost all participants reported that they would keep using the device and were willing to share the device and educational infographics with others, the quotes below are typical of many responses.

"I will keep on following the same protocol and writing down the numbers. I might do it once or twice a week. I will share the machine with my wife." (Male, 68 years)

"I shared the brochure with my neighbors [...]. One of my neighbors is pregnant and has hypertension so I shared the brochure with her." (Female, 65 years)

Acceptability

The majority of participants (33/38) reported that the device was easy to use and convenient, compared for example to having to go to the pharmacy for monitoring, and several mentioned that self-monitoring had become part of their routine. The quotes below illustrate how self-monitoring can be positively incorporated into daily life. 
"The machine is placed next to my bed. It is a part of my life now. It is like the bible now, always next to me." (Female, 60 years)

"Yes, it allowed me to always monitor my blood pressure regularly; I cannot live without it now-either in the evening or in the morning. [The machine] started to feel like a medicine [in terms of timing, adherence and regularity]. [...] I take it with me wherever I go." (Male, 65 years)

Many participants also reported that the use of the device gave them peace of mind and reduced their worries about getting high blood pressure numbers.

"One gets peace of mind when measuring BP with the machine. The machine helped me a lot to have peace of mind mentally and emotionally." (Female, 69 years)

"The machine helped me monitor my blood pressure whenever I want. The numbers are accurate because it is digital [...]. It gives you peace of mind and stability as long as your blood pressure numbers fall within a certain range" (Male, 60 years)

"When I measure my blood pressure and find it normal, I am psychologically at peace. Thank God it is regulated now." (Male, 55 years)

Very few participants (3/38) reported that monitoring their blood pressure made them anxious. They described how they would get nervous while waiting for the device to give them their blood pressure numbers, and would feel relieved when the levels were within the normal ranges.

"When the study is over, I will not look at my blood pressure, it is useless because I will get anxious (Female, 55 years)

Most participants did not report issues with implementing the protocol as required and found the diary easy to use, but some reported difficulties in remembering and taking the required blood pressure measurements, writing down the time of measurement, or entering information in the diary. Some reported getting help from family members to complete the task correctly and a few reported that selfmonitoring was burdensome due to family or work responsibilities. Only a few participants chose to write down additional notes about their feelings and symptoms. The following quotes illustrate some of the reported difficulties with self-monitoring protocol.

"Sometimes I forgot to take my blood pressure, so I got a little bit worried about not following the protocol." (Male, 63 years)

"It was good, but I was so bothered. Because I was busy, and my daughter was reminding me [of taking my blood pressure]. The only thing that was annoying me is the time because I am taking care of my granddaughter who is a 4 months' baby. (Female, 56 years)

Regarding the information provided as part of the educational intervention, participants appeared interested in learning about hypertension, especially those recruited from primary care centers. Patients in 
the self-monitoring group found the infographics to be clear and helpful. Although few participants reported returning to the brochure, many said they acquired knowledge about hypertension and its management, and how to measure blood pressure. Most participants also learned about the importance of measuring blood pressure regularly, which foods are salt-rich, the effect of salt on hypertension, the notion of blood pressure target numbers, and what to do when blood pressure is high. The quotes below illustrate respondents' reports about what they learned.

"I did everything you taught me. I put my hand on the table, back straight, [palm up]. If I had eaten or drunk coffee, I would wait for 30 min before taking my blood pressure." (Female, 76 years)

"I thought that tomato contained salt...but she [the researcher] told me that fresh tomato doesn't contain a lot of salt, it is tomato paste that is high in salt." (Male, 60 years)

"I remember the information about blood pressure numbers...if blood pressure reaches 180 we should call the health center." (Male, 63 years)

Effectiveness: primary and secondary outcomes

Table 1 presents the results of our analysis of changes in blood pressure levels, knowledge scores and medication adherence between standard of care and self-monitoring of blood pressure groups.

Changes in blood pressure levels

Compared with the standard of care group, greater and significant reduction in systolic blood pressure, between baseline and endline, occurred in the self-monitoring group (adjusted mean difference: $-6.3 \mathrm{mmHg} ; 95 \% \mathrm{Cl}[-12.4 ;-0.17], \mathrm{p}=0.04)$. Changes in diastolic blood pressure also showed a greater, but not significant, reduction in the intervention group between baseline and 6-week follow-up (adjusted mean difference: $-1.9 \mathrm{mmHg} ; 95 \% \mathrm{Cl}[-6.34 ; 2.58], \mathrm{p}=0.40$ ). 
Table 1

Systolic and diastolic blood pressure, knowledge score, and medication adherence at baseline and 6 weeks?.

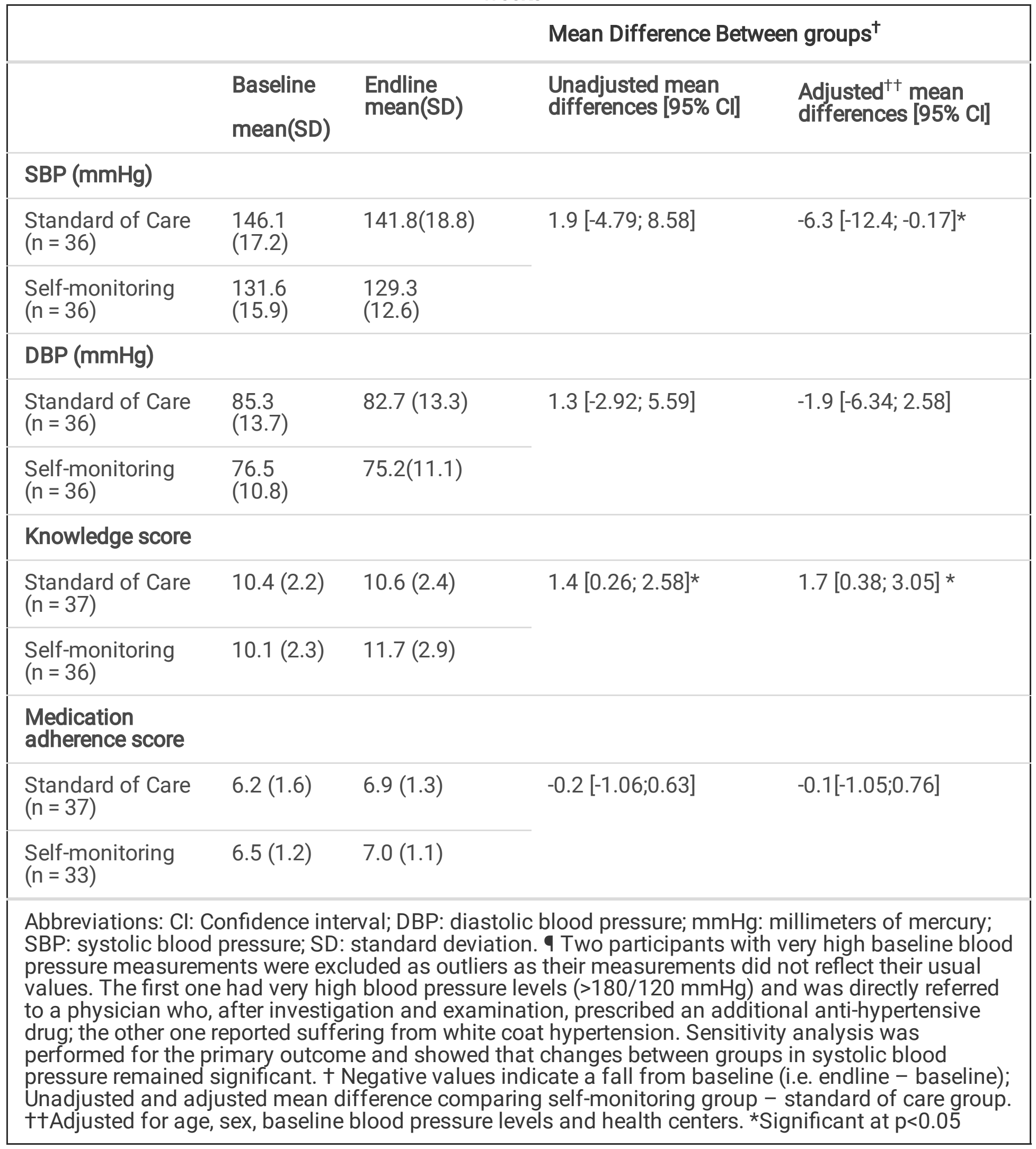

Changes in knowledge scores 
Increase in knowledge scores was significantly higher among the self-monitoring group as compared to the standard of care group in the crude (crude mean difference: +1.4 points; $95 \% \mathrm{Cl}[0.26 ; 2.58], \mathrm{p}=0.02$ ) and adjusted models (adjusted mean difference: +1.7 points; $95 \% \mathrm{Cl}[0.38 ; 3.05]$; $\mathrm{p}=0.01$ ). Improvements in knowledge scores among patients in the monitoring group were highest for questions pertaining to diagnosis of hypertension and to self-monitoring, as shown in table 2.

Table 2. Changes in knowledge score among patients in monitoring group by questions 


\begin{tabular}{|c|c|}
\hline Questions & $\begin{array}{l}\text { Improvement } \\
(\sqrt{ } \text { if } \\
\text { observed })\end{array}$ \\
\hline $\begin{array}{l}\text { Diagnosis of hypertension } \\
\text { easuring your blood pressure is the only way to know if your blood pressure is } \\
\text { gh (T) } \\
\text { ost individuals with increased blood pressure can feel symptoms (F) } \\
\text { is difficult to diagnose hypertension }(F)\end{array}$ & - \\
\hline $\begin{array}{l}\text { Definition of hypertension } \\
\text { ood pressure corresponds to the pressure the blood is exerting against the } \\
\text { tery walls }(T)\end{array}$ & - \\
\hline $\begin{array}{l}\text { Self-monitoring of blood pressure } \\
\text { you want to take your blood pressure and have accurate measurements, you } \\
\text { n borrow or use any device regardless of the cuff size }(F) \\
\text { onitors with a wrist cuff are considered more accurate than those with an arm } \\
\text { ff (F) } \\
\text { u can smoke or drink coffee } 15 \text { min before taking your blood pressure }(F) \\
\text { single high systolic blood pressure reading (between } 140 \text { and } 180 \mathrm{mmHg} \text { ) is } \\
\text { on an immediate cause for alarm and you should not rush immediately to the } \\
\text { spital }(T)\end{array}$ & $\begin{array}{l}\sqrt{ } \\
\sqrt{ }\end{array}$ \\
\hline $\begin{array}{l}\text { Complications of hypertension } \\
\text { creased blood pressure can cause kidney failure, if left untreated (T) }\end{array}$ & - \\
\hline $\begin{array}{l}\text { Understanding hypertension risk factors and its lifestyle and medication treatr } \\
\text { sk factors of hypertension cannot be changed (F) } \\
\text { ood pressure could rise temporarily due to stress, exercise, or intake of salt (T) }\end{array}$ & $\sqrt{ }$ \\
\hline $\begin{array}{l}\text { Management of hypertension } \\
\text { u can stop, reduce, or change medications without physician consultation } \\
\text { pen your blood pressure readings are normal }(F) \\
\text { sing weight usually lowers your blood pressure }(T) \\
\text { ercising every day may lower blood pressure in the long term }(T)\end{array}$ & $\sqrt{ }$ \\
\hline
\end{tabular}


pu should take your blood pressure medications only when you are feeling high ood pressure symptoms $(F)$

you take your anti-hypertensive medicine regularly, there is no need to crease your salt intake $(F)$

\section{Medication and lifestyle changes}

We found no major improvement in medication adherence in our study population and no statistical difference in adherence scores between groups. Indeed, the mean change in Morisky scores between baseline and endline did not differ between the intervention and the standard of care groups (adjusted mean difference: -0.1 point; $95 \% \mathrm{Cl}[-1.05 ; 0.76], \mathrm{p}=0.75$ ) (Table 1 ). Also, over the 6-week study period, changes in types, numbers, or dosages of prescribed antihypertensive medications did not significantly differ between groups (change in medications in self-monitoring group $=22 \%$ vs. standard of care group $=14 \% ; p=0.33)$.

Almost half of the self-monitoring group reported making one or more changes in their lifestyle, such as increasing physical activity, decreasing in salt intake, reducing weight, or starting a healthy diet, compared to only a quarter among the standard of care group ( $44 \%$ vs. $24 \%$ ). The difference between groups was statistically significant after adjusting for age, sex, baseline blood pressure levels and health centers (Adjusted odds ratio $=3.3 ; 95 \% \mathrm{Cl}[1.01 ; 11.16], \mathrm{p}=0.04$ ).

Interestingly, our qualitative data revealed that, while patients acknowledged the importance of medication adherence and lifestyle changes, they were concerned about all the structural barriers that they had to overcome to adopt these healthy choices including the financial constraints to purchase medications and healthy foods, the unavailability of free recreational facilities, and the constant political and economic stress in their lives.

"I went to a dietitian but I did not continue seeing her as it was not affordable to follow the diet she prescribed." (Female, 69 years)

"I have no time. I have my kids. Sport is for people who have nothing to worry about. To be able to exercise you should be mentally and financially relaxed."(Male, 44 years)

"I had to stop the medication as it was really expensive. I would rather spend the money on my kids than buy the medication. My kids are more important than my health. My faith is in God." (Female, 59 years)

\section{Discussion}


To our knowledge, this is the first study in a low- and middle-income country, and in the Arab region, that explores the feasibility, acceptability, and potential effectiveness of an intervention designed to improve blood pressure levels through self-monitoring and education. Our mixed-method approach also helps better understand the experiences and perceptions of the participants.

The analysis indicates that our study protocol was feasible, with good recruitment, retention, and measurement adherence rates. Refusals to participate were explained by lack of time or interest, or other priorities, while reasons for dropping out were due to logistic constraints such as long commuting time to reach the center. The short follow-up period, the reimbursement of transportation, and the reminders prior to the interview, likely contributed to the high retention among both groups. The fact that those in the monitoring group could keep the monitoring device after the study was completed represented a further incentive among these participants. We believe however that the main contributors to recruitment and retention were the continuity of care that all participants received and their trust in the providers at the health centers. Thus, we think that the success of monitoring interventions would be greater in settings that offer continuity of care and foster trust in providers.

Most participants valued the monitors and understood the importance of regularly measuring blood pressure, stating that it gave them peace of mind and security. Only a few participants made spontaneous notes on their feelings or recorded additional blood pressure measurements. We believe that, had the resources been available for a longer follow-up, participants would have been more encouraged to take their blood pressure outside the fixed protocol and to note their experiences. It is also likely that patients from low social strata are not accustomed to being asked for their opinions, nor are they used to making notes about their experiences and hence were reluctant to volunteer to express themselves (37). Moreover, our situation analysis had suggested that monitoring of blood pressure could be a source of anxiety which leads some patients to seek unnecessary care at health care facilities or emergency departments. The results of this study show that only a small proportion of the participants reported feeling anxious and/or sought additional care due to blood pressure readings. We attribute this to the fact that our intervention included an educational component to explain the meaning of blood pressure numbers, what to do when blood pressure is elevated, and when it is appropriate to seek care.

Overall, it is remarkable that despite the small study sample, we were able to demonstrate that the intervention showed promising results in reducing blood pressure. The decrease in blood pressure levels among the self-monitoring group is in line with the results of another study conducted in two low- and middle-income countries: Honduras and Mexico (23). The educational materials we developed were easy to understand, as indicated by participants' improved knowledge scores. The highest improvements were around questions related to the correct measurement of blood pressure, and can be attributed to the hands-on training on measurement and to the thorough one-on-one educational sessions about hypertension management conducted at the beginning of the study. The fact that improvements in knowledge and in blood pressure levels were greater in the intervention group suggests that it is not the ownership of a device nor the frequency of blood pressure monitoring at baseline (which were statistically similar among both groups at baseline) but the educational intervention and the engagement 
of patients in the monitoring process, that account for these improvements. Our intervention included a one-on-one session to train patients to measure their blood pressure, explanations about the meaning of blood pressure readings, a diary to record regular blood pressure measurements, and follow-ups. We believe that these elements contributed to better knowledge, stronger motivation, and higher engagement of patients than the standard hypertension care provided at the health centers to patients. Providing information along with devices should be part of future interventions. It is however, infrequently done, and the situation analysis we conducted showed that many health providers do not explain the meaning of blood pressure readings and the recommended blood pressure targets, and do not show to patients how to use monitoring devices and how to ensure accurate blood pressure monitoring. Our study showed a significant higher change in reported healthy lifestyle habits among patients from the self-monitoring group as compared to the standard of care one. Over the 6-week study period, changes in types, numbers, or dosages of prescribed antihypertensive medications as well as in adherence to medication did not statistically differ between groups; and medication adherence levels did not increase in our study possibly due to structural and financial obstacles as reported in other studies $(38,39)$. We can thus speculate that the improvement in lifestyle habits might partly explain the greater reduction in blood pressure levels observed among the intervention group. We are unable to distinguish the effect of each component of the intervention on blood pressure levels, but we believe that it is the synergistic effect of the health education and the self-monitoring device on patient understanding and engagement that contributed to patients' improved blood pressure levels (22).

This study has implications for thinking about similar interventions in other low- and middle-income countries. Contrary to studies demonstrating the differential impact of health interventions which are often taken up by the better off $(40,41)$, improvements in blood pressure numbers and lifestyle habits were reported among all our study sample which included a majority of participants from low to middle socio-economic status. Interestingly, access to blood pressure monitors outside the study was generally high among our respondents, despite the relatively high cost of blood pressure monitors (around \$50). We found that among 58 patients from lower socio-economic groups-irrespective of whether they were assigned to control or intervention group-nearly half (27) reported having access to a blood pressure machine; some had purchased it, others received it as a gift, and others reported borrowing machines from their neighbors or family members. Our study highlights the importance of health information and motivation towards monitoring, over and above the mere access to devices. This is consistent with the observation that hypertensive patients from low socio-economic groups face barriers to controlling blood pressure beyond simply having access to a blood pressure monitor (25). These obstacles include limited access to health care, difficulties in communicating with health care providers, financial constraints in purchasing medications or following a healthy diet $(42,43)$. Indeed, many of our participants mentioned structural barriers and stressful lives as obstacles to adopting healthier choices.

Uncontrolled blood pressure is a major public health issue in the Arab world, regardless of wealth or level of development (11). The cultural similarities between Lebanon and other Arab countries, and the diversity in socioeconomic status, modes of health care delivery, and degree of medicalization in Lebanon, imply that the results of the project are relevant to the region as a whole. Similar strategies 
incorporating self-monitoring of blood pressure with education and counselling have great potential in optimizing hypertension management in both high- and middle-income Arab countries.

\section{Limitations}

This proof of concept study was intended to examine feasibility, acceptability, and potential impact of self-monitoring of blood pressure and educational sessions on blood pressure levels. It was not powered to conduct statistical analyses of effectiveness. Moreover, this study used self-reported data to measure lifestyle changes which may reflect social-desirability bias. The potential for such bias is likely reduced by the use of qualitative data to complement quantifiable responses, as the texts spontaneously provided by respondents are consistent with real efforts to change lifestyles. Another limitation is the short follow-up period of 6 weeks; a longer study-period with additional interviews and counselling would provide more robust results and assess the sustainability of the intervention over time.

\section{Conclusions}

This proof-of-concept study demonstrated good feasibility, acceptability, and potential effectiveness of a strategy combining self-monitoring of blood pressure with educational sessions in reducing blood pressure levels, improving knowledge scores, and promoting lifestyle changes among hypertensive patients. These results should be tested on a larger sample with a longer follow-up and with the addition of a counseling component. Overall, our study suggests that monitoring and education may improve hypertension management in Lebanon as well as in other Arab countries of the region.

\section{Abbreviations}

BP: Blood pressure

Cl: confidence interval

DBP: Diastolic blood pressure

IQR: interquartile range

LMICs: Low and middle-income countries

mmHg: millimeters of mercury

OR: Odd Ratio

SBP: Systolic blood pressure

SD: Standard deviation

\section{Declarations}




\section{- Ethics approval and consent to participate}

The study was approved by the Institutional Review Board of the American University of Beirut (SBS2017-0612). Participants were informed about the purpose, content, and procedure of the study and all patients provided written informed consent at the beginning of the study. The trial was registered retrospectively in the ISRCTN registry (number 16450193) on April 3, 2020. http://www.isrctn.com/ISRCTN16450193

\section{- Consent to publish}

Not applicable.

\section{- Availability of data and materials}

The datasets generated and analyzed during the current study are not publicly available as, in a proof of concept study such as ours, the datasets are small and we hope to expand them over time. However, the datasets are available from the corresponding author on reasonable request. Our data are de-identified and do not include any direct or indirect identifiers; they thus cannot compromise anonymity or confidentiality. Participants were informed that the de-identified data will be used for research purposes and written consent was obtained.

\section{- Competing interests}

The authors declare that they have no competing interests.

\section{- Funding}

This work was funded by a grant (027562-746846-0205) from the Harvard Medical School Center for Global Health Delivery in Dubai. The funders had no role in study design, data collection, analysis and interpretation, decision to publish, or preparation and writing of the manuscript.

\section{- Acknowledgments}

We would like to thank Ms. Sandra Kahwaji and Ms. Zahra El-Amine for their contribution to the fieldwork and to the data collection process. We also thank all the patients who participated in this study as well as all the managers and health care providers who facilitated access to the selected health centers and helped in the logistics of this study.

- Authors' contributions

CA (Akl) and NR drafted the paper and conducted data collection, analysis and interpretation. CA (Akik) supervised the work, contributed to data analysis, interpretation and writing. CMO and HG designed the 
study and supervised the work. WF contributed to the study design. $\mathrm{EH}, \mathrm{GH}, \mathrm{KB}, \mathrm{MR}, \mathrm{SM}, \mathrm{AG}$ and SO critically reviewed and contributed to the study design and proposal and facilitated data collection and recruitment. All authors critically reviewed the paper.

\section{References}

1. Danaei G, Finucane MM, Lin JK, Singh GM, Paciorek CJ, Cowan MJ, et al. National, regional, and global trends in systolic blood pressure since 1980: systematic analysis of health examination surveys and epidemiological studies with 786 country-years and 5.4 million participants. Lancet. 2011;377(9765):568-77.

2. Ezzati M, Riboli E. Behavioral and Dietary Risk Factors for Noncommunicable Diseases. N Engl J Med. 2013;369(10):954-64.

3. Lim SS, Vos T, Flaxman AD, Danaei G, Shibuya K, Adair-Rohani H, et al. A comparative risk assessment of burden of disease and injury attributable to 67 risk factors and risk factor clusters in 21 regions, 1990-2010: a systematic analysis for the Global Burden of Disease Study 2010. Lancet (London, England). 2012;380(9859):2224-60.

4. Global regional. and national age-sex specific all-cause and cause-specific mortality for 240 causes of death, 1990-2013: a systematic analysis for the Global Burden of Disease Study 2013. Lancet (London, England). 2015;385(9963):117-71.

5. Mouhtadi BB, Kanaan RMN, Iskandarani M, Rahal MK, Halat DH. Prevalence, awareness, treatment, control and risk factors associated with hypertension in Lebanese adults: A cross sectional study. Global cardiology science practice. 2018;2018(1):6.

6. Yusufali AM, Khatib R, Islam S, Alhabib KF, Bahonar A, Swidan HM, et al. Prevalence, awareness, treatment and control of hypertension in four Middle East countries. J Hypertens. 2017;35(7):145764.

7. Chow CK, Gupta R. Blood pressure control: a challenge to global health systems. The Lancet. 2019;394(10199):613-5.

8. Mills KT, Bundy JD, Kelly TN, Reed JE, Kearney PM, Reynolds K, et al. Global Disparities of Hypertension Prevalence and Control: A Systematic Analysis of Population-Based Studies From 90 Countries. Circulation. 2016;134(6):441-50.

9. Zhou B, Danaei G, Stevens GA, Bixby H, Taddei C, Carrillo-Larco RM, et al. Long-term and recent trends in hypertension awareness, treatment, and control in 12 high-income countries: an analysis of 123 nationally representative surveys. The Lancet. 2019;394(10199):639-51.

10. Geldsetzer P, Manne-Goehler J, Marcus M-E, Ebert C, Zhumadilov Z, Wesseh CS, et al. The state of hypertension care in 44 low-income and middle-income countries: a cross-sectional study of nationally representative individual-level data from $1 \cdot 1$ million adults. The Lancet. 2019;394(10199):652-62. 
11. Akl C, Akik C, Ghattas H, Makhlouf Obermeyer C. The Cascade of Care in managing hypertension in the Arab world: A systematic assessment of the evidence on awareness, treatment and control. BMC Public Health. 2020;In press.

12. World-Bank. How we classify countries. [Available from: http://data.worldbank.org/about/countryclassifications.

13. Sarki AM, Nduka CU, Stranges S, Kandala NB, Uthman OA. Prevalence of Hypertension in Low- and Middle-Income Countries: A Systematic Review and Meta-Analysis. Medicine. 2015;94(50):e1959.

14. Noubani A. Prevalence. Awareness, and Control of Hypertension in Greater Beirut Area, Lebanon. International journal of hypertension. 2018;2018:1-15.

15. Stergiou GS, Kollias A, Zeniodi M, Karpettas N, Ntineri A. Home blood pressure monitoring: primary role in hypertension management. Curr Hypertens Rep. 2014;16(8):462.

16. Turner JR, Viera AJ, Shimbo D. Ambulatory blood pressure monitoring in clinical practice: a review. Am J Med. 2015;128(1):14-20.

17. Aekplakorn W, Suriyawongpaisal P, Tansirisithikul R, Sakulpipat T, Charoensuk P. Effectiveness of Self-Monitoring Blood Pressure in Primary Care: A Randomized Controlled Trial. Journal of primary care community health. 2016;7(2):58-64.

18. Fahey T, Schroeder K, Ebrahim S. Educational and organisational interventions used to improve the management of hypertension in primary care: a systematic review. The British journal of general practice: the journal of the Royal College of General Practitioners. 2005;55(520):875-82.

19. Clark C, Smith L, Glynn L, Cloutier L, Clark O, Taylor R, et al. Changes in blood pressure in patients with hypertension receiving usual care in randomised controlled trials. Findings from a systematic review and meta-analysis. J Hypertens. 2015;33:e85.

20. Coulter A. Patient. engagement-what works? The Journal of ambulatory care management. 2012;35(2):80-9.

21. Mc Namara KP, Versace VL, Marriott JL, Dunbar JA. Patient engagement strategies used for hypertension and their influence on self-management attributes. Family practice. 2014:cmu026.

22. Tucker KL, Sheppard JP, Stevens R, Bosworth HB, Bove A, Bray EP, et al. Self-monitoring of blood pressure in hypertension: A systematic review and individual patient data meta-analysis. PLoS Med. 2017;14(9):e1002389.

23. Piette JD, Datwani H, Gaudioso S, Foster SM, Westphal J, Perry W, et al. Hypertension management using mobile technology and home blood pressure monitoring: results of a randomized trial in two low/middle-income countries. Telemedicine e-Health. 2012;18(8):613-20.

24. Anderson C, Dadabhai S, Damasceno A, Dzudie A, Islam SMS, Kamath D, et al. Home blood pressure management intervention in low-to middle-income countries: protocol for a mixed methods study. JMIR research protocols. 2017;6(10):e188.

25. Yi SS, Tabaei BP, Angell SY, Rapin A, Buck MD, Pagano WG, et al. Self-blood pressure monitoring in an urban, ethnically diverse population: a randomized clinical trial utilizing the electronic health record. Circulation: Cardiovascular Quality Outcomes. 2015;8(2):138-45. 
26. Yamout R, Adib SM, Hamadeh R, Freidi A, Ammar W. Screening for cardiovascular risk in asymptomatic users of the primary health care network in Lebanon, 2012-2013. Prev Chronic Dis. 2014;11:E120.

27. Eldridge SM, Chan CL, Campbell MJ, Bond CM, Hopewell S, Thabane L, et al. CONSORT 2010 statement: extension to randomised pilot and feasibility trials. bmj. 2016;355:i5239.

28. High Blood Pressure National Heart, Lung and Blood Institute; [Available from: https://www.nhlbi.nih.gov/health-topics/high-blood-pressure.

29. High Blood Pressure. American Heart Association; [Available from: https://www.heart.org/en/healthtopics/high-blood-pressure.

30. Chinn D, McCarthy C. All Aspects of Health Literacy Scale (AAHLS): developing a tool to measure functional, communicative and critical health literacy in primary healthcare settings. Patient Educ Couns. 2013;90(2):247-53.

31. Morisky DE, Ang A, Krousel-Wood M, Ward HJ. Predictive validity of a medication adherence measure in an outpatient setting. The Journal of Clinical Hypertension. 2008;10(5):348-54.

32. Lee GK, Wang HH, Liu KQ, Cheung Y, Morisky DE, Wong MC. Determinants of medication adherence to antihypertensive medications among a Chinese population using Morisky Medication Adherence Scale. PloS one. 2013;8(4):e62775.

33. Matar D, Frangieh AH, Abouassi S, Bteich F, Saleh A, Salame E, et al. Prevalence, awareness, treatment, and control of hypertension in Lebanon. Journal of clinical hypertension (Greenwich Conn). 2015;17(5):381-8.

34. Lancaster GA, Dodd S, Williamson PR. Design and analysis of pilot studies: recommendations for good practice. Journal of evaluation in clinical practice. 2004;10(2):307-12.

35. Waterlander WE, Steenhuis IH, de Boer MR, Schuit AJ, Seidell JC. The effects of a $25 \%$ discount on fruits and vegetables: results of a randomized trial in a three-dimensional web-based supermarket. International Journal of Behavioral Nutrition Physical Activity. 2012;9(1):11.

36. Alshurafa M, Briel M, AkI EA, Haines T, Moayyedi P, Gentles SJ, et al. Inconsistent definitions for intention-to-treat in relation to missing outcome data: systematic review of the methods literature. PLoS One. 2012;7(11).

37. Obermeyer CM. Risk, uncertainty, and agency: culture and safe motherhood in Morocco. Med Anthropol. 2000;19(2):173-201.

38. Najimi A, Mostafavi F, Sharifirad G, Golshiri P. Barriers to medication adherence in patients with hypertension: A qualitative study. Journal of education and health promotion. 2018;7.

39. Burnier M, Egan BM. Adherence in hypertension: a review of prevalence, risk factors, impact, and management. Circulation research. 2019;124(7):1124-40.

40. Phelan JC, Link BG, Tehranifar P. Social conditions as fundamental causes of health inequalities: theory, evidence, and policy implications. Journal of health social behavior. 2010;51(1_suppl):28-40.

41. Rose G. Sick individuals and sick populations. Int J Epidemiol. 2001;30(3):427-32. 
42. Pampel FC, Krueger PM, Denney JT. Socioeconomic disparities in health behaviors. Annual review of sociology. 2010;36:349-70.

43. Laraia BA, Leak TM, Tester JM, Leung CW. Biobehavioral factors that shape nutrition in low-income populations: a narrative review. Elsevier; 2017.

\section{Supplementary Files}

This is a list of supplementary files associated with this preprint. Click to download.

- supplement1.docx

- supplement2.docx

- supplement3.docx

- supplement4.docx 